\title{
The Dynamic Behavior of Opossum Shrimps
}

\author{
Seiichi Sudo ${ }^{\text {a, }}$, Kyohei Hoshika ${ }^{\text {a }}$, Tetsuya Yano ${ }^{a}$ and Yoshiaki Shimazaki ${ }^{\text {a }}$ \\ ${ }^{\mathrm{a}}$ Faculty of Systems Science and Technology, Akita Prefectural University, Japan
}

\begin{abstract}
The dynamic behavior of small shrimp-like creature is analyzed by a digital high speed video camera system. Test small aquatic creature is opossum shrimp, Archaeomysis kokuboi Ii. Some movement models of opossum shrimps are observed. The details of the motion of swimming legs and flow fields are revealed experimentally. Flow visualization around tethered opossum shrimp is also performed by slow shutter photographic technique.
\end{abstract}

Index Terms - Bio-fluid Dynamics, Swimming, Flow visualization, Opossum Shrimps

\section{INTRODUCTION}

A wide variety of living creatures live on this planet. Most animals move about using legs, wings, or fins. The motion of animals in the environmental fluids most dictates the morphology and physiology of animals. The dynamic behavior of animals has long attracted the attention of biologists, engineers, and other scientists for many years [1]. Recent advances in the integrative and comparative studies of animal locomotion have been reviewed by Dickinson et al. [2].

The swimming motions of aquatic animals are remarkable and fascinating. With increasing interest in swimming robots, extensive investigations have been conducted on the interactions of aquatic animals with their surrounding fluid medium [1]. In our previous paper, the surface roughness of some fish scales was measured by a three-dimensional, optical shape measuring system to provide information related to drag reduction [3]. The swimming behavior of diving beetles was also analyzed by a digital high speed video camera system, and a wireless diving beetle robot propelled by an alternating magnetic field was developed [4]. The swimming behavior of small aquatic creatures was analyzed to make a miniature swimming robot [5]. In spite of many investigations, there still remains a wide unexplored domain.

In this paper, the dynamic behavior of opossum shrimp, Archaeomysis kokuboi $\mathrm{Ii}$, is analyzed with the digital high speed video camera system. The free swimming behavior of opossum shrimp is analyzed, first. Then, the flow field around tethered opossum shrimp is analyzed, and it is visualized by the slow shutter photographic technique. The details of swimming modes and flow patterns of opossum shrimp are revealed experimentally.

\section{OPOSSUM SHRIMP}

In this paper, the dynamic behavior of opossum shrimp was studied by the optical measurement method. The opossum shrimps are a member of the crustacean order Mysidacea. Most of the nearly 1000 known species live in the sea, and they are mostly $10 \mathrm{~mm}$ to $30 \mathrm{~mm}$ long. The opossum shrimps are shrimp-like animals that are characterized by a marsupium within which the entire larval development takes place. Figure 1 shows the photograph of a wild opossum shrimp. The name opossum shrimp derives the female's brood pouch. The brood pouch is composed of two, three or seven pairs of lamellae attached to the thoracic limbs. The uropods consist of lamellar endopods and exopods forming a tail fan with the telson. The endopods usually possess a statocyst. The telson is always wider at the base than at the apex. The abdominal legs never have chelae. The shield-like carapax covers the greater part of the cephalothorax, but is not attached to it in the last thoracal segments. Opossum shrimp, Archaeomysis kokuboi Ii, has a slender body as shown in Fig.1. Its transparent body has occasional brown pigmentation. The large, stalked eyes are conspicuous. The two pairs of antennae are long and biramous.

In this experiment, the swimming behavior of opossum shrimp in seawater, and the jumping behavior in the air were examined with the high speed video camera system. The flow field around the tethered opossum shrimp was visualized by the slow shutter photographic technique, because the knowledge of the velocity field is the most important information needed in the analysis of fluid flow problem.

\section{EXPERIMENTAL METHOD}

Figure 2 shows the schematic diagram of the experimental apparatus to study swimming characteristics of opossum shrimp. The experimental apparatus consists of the seawater container system and the optical measurement system. Two kinds of containers were used for swimming analysis and flow

\footnotetext{
* Corresponding author: Ebinokuchi 84-4, Yurihonjo City, Akita
} 015-0055, Japan, E-mail:sudo@akita-pu.ac.jp 


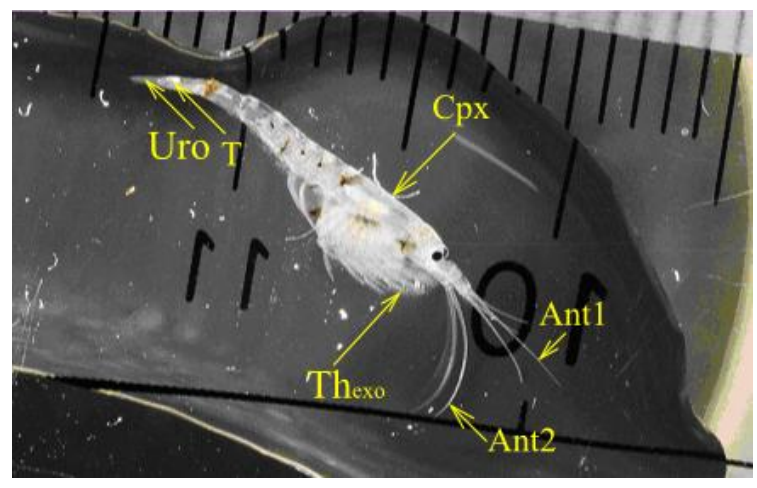

(a) whole body in lateral view

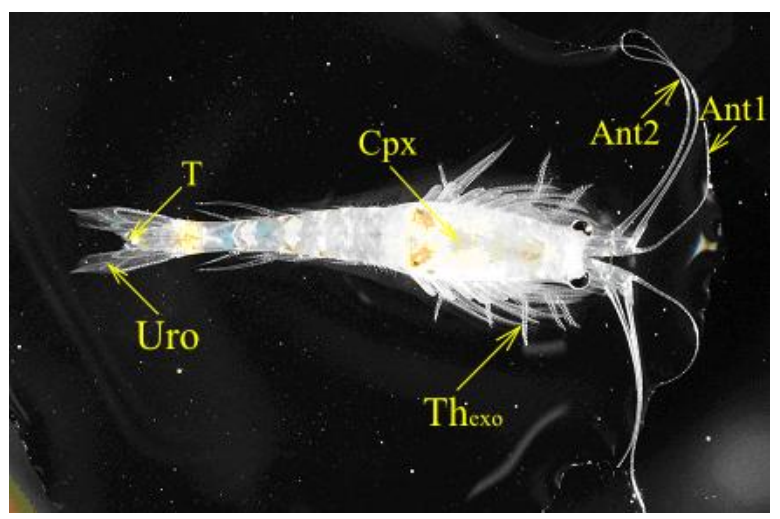

(b) whole body in dorsal view

Fig. 1. Photographs of opossum shrimp. Ant1: $1^{\text {st }}$ antennae, Ant2: $2^{\text {nd }}$ antennae, Cpx: Carapax, $\mathrm{Th}_{\mathrm{exo}}$ : expodite of thoracopod, $\mathrm{T}$ : Telson, Uro: Uropods.

visualization, a rectangular transparent acrylic plastic container and a cylindrical glass container. In the experiment, the rectangular container was filled with mixture of seawater and mud for visualization of flow induced by the motion. Free swimming and tethered swimming of opossum shrimp were observed optically with the high speed video camera. A series of digitally recorded frames of swimming behavior of opossum shrimp were analyzed using a personal computer. In the experiment of tethered swimming analysis, a live opossum shrimp was pasted up on human hair or rigid needle with the adhesive. The motion of each part of opossum shrimp body was recorded by the high speed video camera. In the experiment for flow visualization, the cylindrical container was partially filled with seawater. The powder of chaff was scattered on the water surface. Powder of chaff, which plays the role of a flow tracer, was scattered on the water surface. Movement of powder on the water surface and in the water was photographed as a flow. Photographs of flow field were taken with $35 \mathrm{~mm}$ camera as shown in Fig. 2.

\section{EXPERIMENTAL RESULTS AND DISCUSSION}

\section{A. Flow field around Tethered Opossum Shrimp}

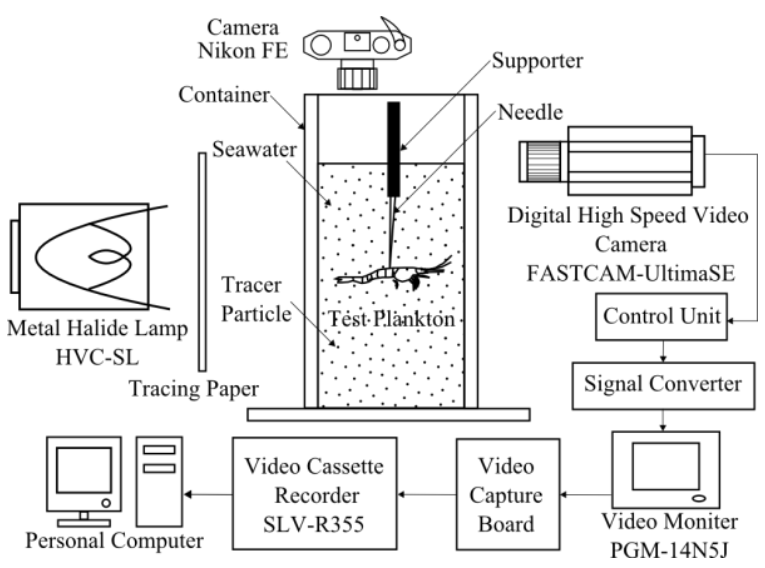

Fig. 2. Block diagram of experimental apparatus.

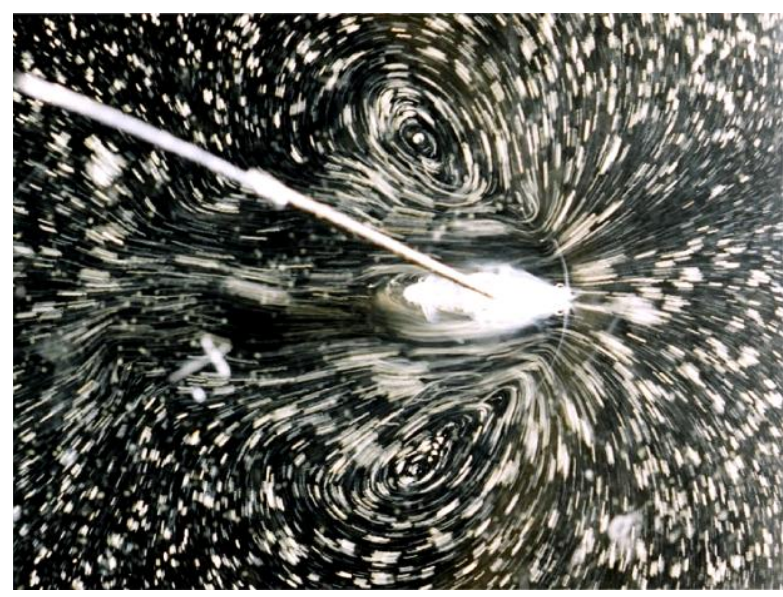

Fig. 3. Flow visualization around a tethered opossum shrimp in dorsal view.

Opossum shrimps have swimming legs in their cephalothoraxes. In ordinary cruise swimming, they row their legs to generate forward thrust. In general, the right- and left-legs beat almost synchronously. The driving force is generated by the fluid dynamic drag of the swimming legs, i.e., leg motion generates backward flow. Figure 3 shows the photograph of visualized flow field around the tethered opossum shrimp in dorsal view. A stream is generated by beat motion of swimming legs of the opossum shrimp. The opossum shrimp swims forward, by pressing the swimming legs backwards against water. This photograph was taken with a $35 \mathrm{~mm}$ camera, shutter speed at $1 / 15 \mathrm{~s}$. The knowledge of the travel distance of particles from Fig. 3 and the shutter speed enables us to find the velocity distribution of the flow field. Figure 4 shows the velocity distribution of the flow field generated by the leg strokes of the tethered opossum shrimp. In Fig. 4, the arrows show the velocity vectors of flow. A vector arrow shows both magnitude and direction of flow velocity. The length of arrow corresponds to the magnitude of velocity, and the direction of arrow corresponds to the flow direction. The scale vector is described in the upper part of Fig. 4. It can be seen from Fig. 4 that the flow is generated from the head to tail of 


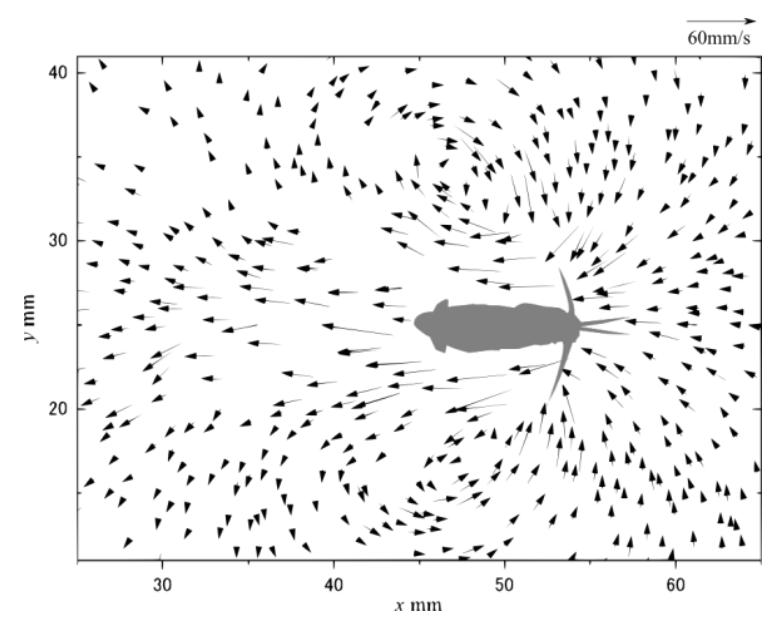

Fig. 4. Velocity distribution around a tethered opossum shrimp.

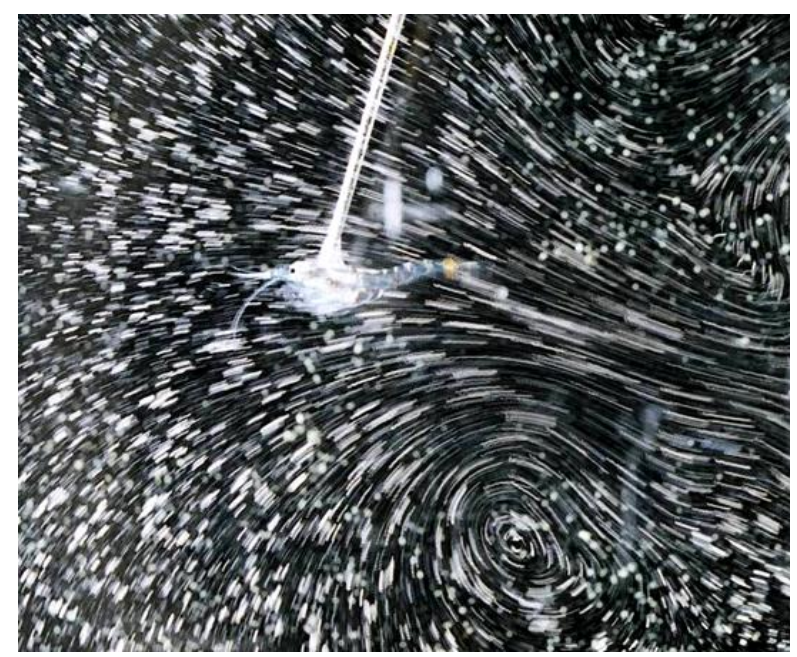

Fig. 5. Flow visualization around a opossum shrimp.

opossum shrimp, that is, the swimming legs press backwards against the seawater, which pushes the opossum shrimp forwards. In this experiment, the maximum flow velocity was about $65 \mathrm{~mm} / \mathrm{s}$ in the neighborhood of opossum shrimp tail.

Figure 5 shows the photograph of visualized flow field around the tethered opossum shrimp in lateral view. In Fig. 5, the shutter speed was set as 1/8 s. The opossum shrimp body is super-paddle pushing a mass of water back and down so that the opossum shrimp is buoyed up and propelled forwards. In this case, the forward thrust is held back by the resisting force of drag. In general, the thrust is described as follows [6];

$$
T=\frac{1}{2} \rho S C_{D} U^{2}+\alpha \rho V \frac{d U}{d t}+m \frac{d U}{d t}
$$

where $\rho$ is the density of fluid, $C_{D}$ is the drag coefficient for motion, $U$ is the instantaneous velocity of the body, $\alpha$ is the added-mass coefficient that depends upon the orientation of the body, $S, V$, and $m$ are, respectively, the surface area, volume and mass of the body, and $t$ is time. Figure 5 enables us to visualize the

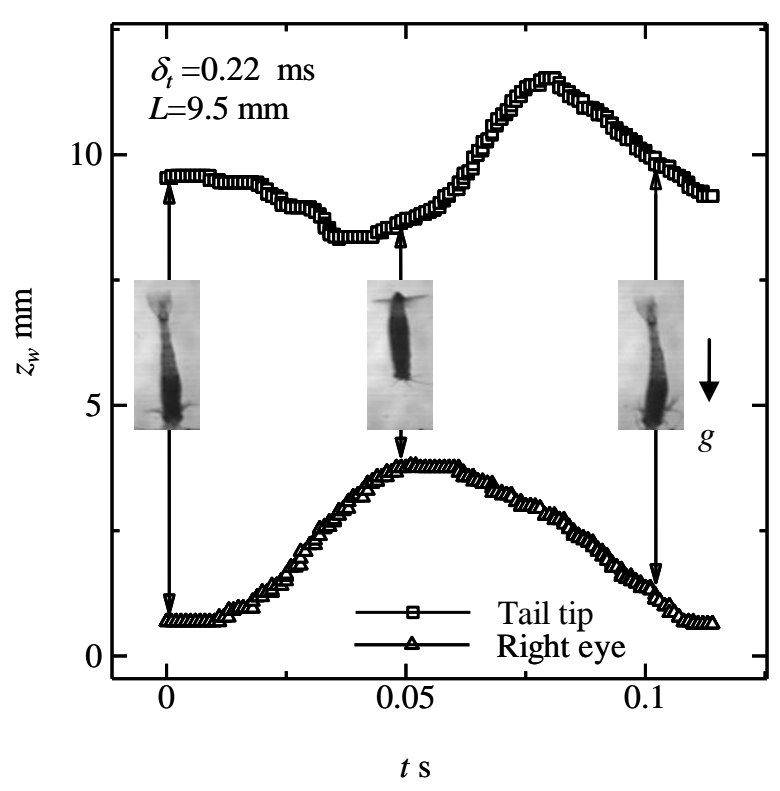

Fig. 6. Backward swimming by the telson and forward swimming.

fact that the thrust is produced by oscillation of swimming legs.

\section{B. Swimming with Telson Fin}

Opossum shrimps show backward swimming sometimes. The opossum shrimp rows water in the forward by the telson fin. The telson fin presses forwards against the water and this pushes the opossum shrimp backwards. Figure 6 shows backward swimming by the telson and forward swimming by swimming legs. Two plots show the position of a tail tip ( $\square$ ) and a right eye $(\Delta)$. In Fig. $6, z_{w}$ is one-dimensional fixed coordinate system located on the container bottom, $\delta_{t}$ is the time interval in the data sampling of opossum shrimp motion, $L$ is the body length of test opossum shrimp, and $g$ is the gravity. Three photographs show the states of opossum shrimp corresponding to the arrowed data. In the beginning stage of Fig. 6, the uropods are formed as shallow caps to increase the hydrodynamic drag and inertial force during the power stroke. The tail tip of opossum shrimp moves downward. And the trajectory of eyes moves upward slowly. In the final stage of Fig. 6, the opossum shrimp rows swimming legs, and moves downward.

However, the opossum shrimp performs rapid fanning motion of the tail occasionally. This swimming mode is used as an escape reaction from predators.

Figure 7 shows a sequence of photographs showing the tethered swimming behavior of opossum shrimp. In this experiment, the live opossum shrimp was glued to a human hair with the adhesive. Since the opossum shrimp was in a flexible state, swimming movement was possible for slight distance. The ventral view of the opossum shrimp shows that the uropods spread out to form a fan during the flip. The fanning of the uropods generates a strong downward flow. This flow pushes up the body of opossum shrimp upwards. Figure 8 shows 


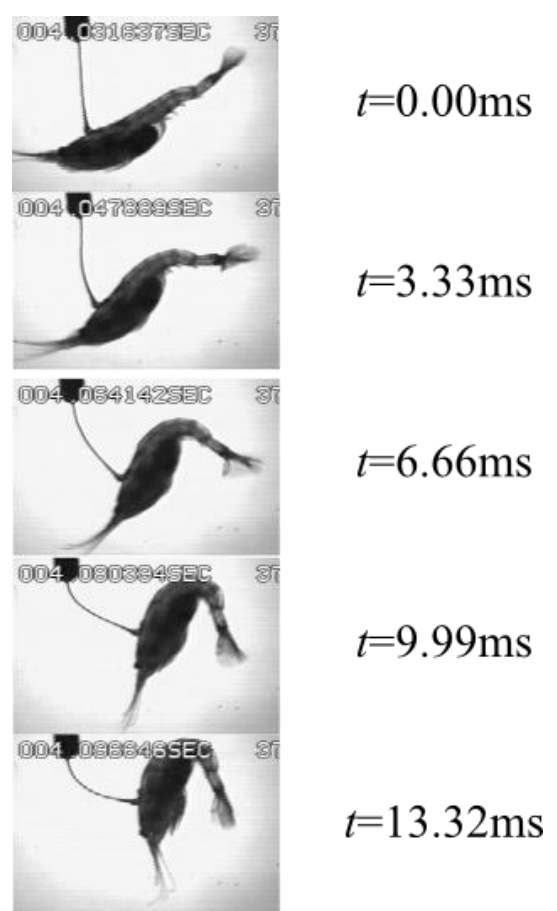

Fig. 7. High-speed frames of escape locomotion with the uropods.

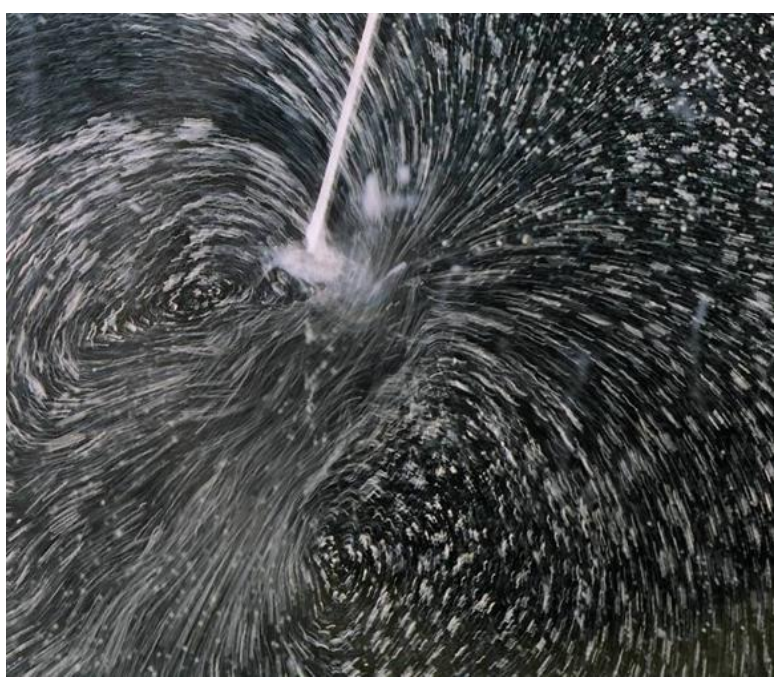

Fig. 8. Flow visualization around the tethered opossum shrimp.

the visualization photograph of flow field produced by the fanning of the uropods. In this case, the thrust is equivalent to jet propulsion [1].

$$
T=Q_{m} U
$$

where $Q_{m}$ is the mass flow rate, and $U$ is the velocity of downward flow. In this experiment, we can estimate the thrust produced by jet propulsion. Putting $Q_{m}=3.3 \times 10^{3}$ $\mathrm{kg} / \mathrm{s}\left(\rho=1040 \mathrm{~kg} / \mathrm{m}^{3}\right.$, and volume flow rate $V_{m}=$ $3.2 \times 10^{-6} \mathrm{~m}^{3} / \mathrm{s}$ ) and $U=1.8 \mathrm{~m} / \mathrm{s}$ (in this calculation, $U$ is the velocity of uropods movement), $T=5.94 \times 10^{-3} \mathrm{~N}$.

\section{Opossum Shrimp Jumping in the Air}

Opossum shrimps showed sudden swimming as before. Such escape swimming of the opossum shrimp

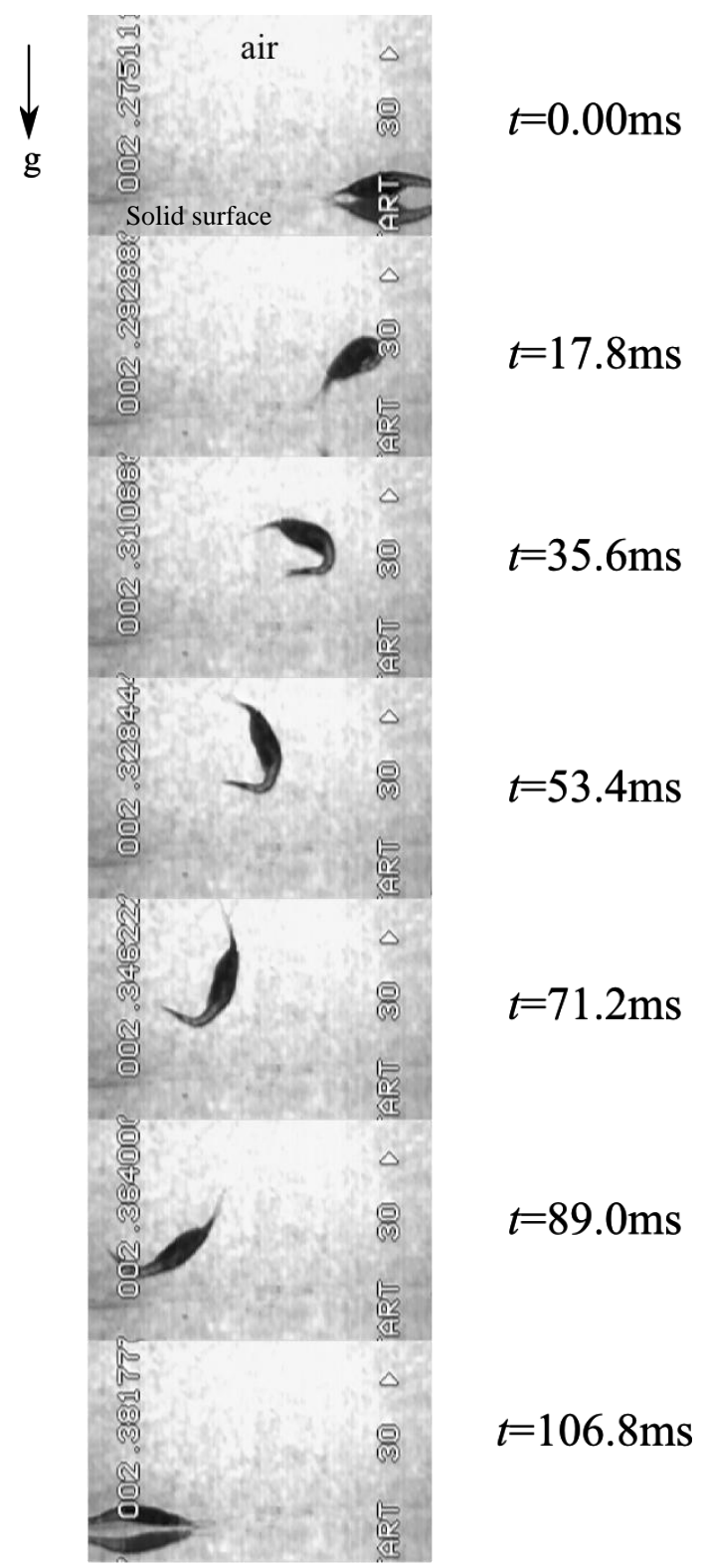

Fig. 9. Photographs of a sequence of stages on the opossum shrimp jumping in the air.

was the result of a rapid flexion of the abdomen. Opossum shrimps utilize a powerful tail-flip in escape from predation. Next, we examine the jump motion of opossum shrimp in the air to determine the kinetic energy.

Figure 9 shows a sequence of photographs showing the free jump of opossum shrimp in the air. In this experiment, the opossum shrimp was laid on the dry acrylic plastic plate. Escape action of the opossum shrimp was recorded with the high-speed video camera system. In this action, elastic energy saved in opossum shrimp flexion is transformed into potential energy. If we neglect air resistance, the maximum jump height in Fig. 9 is equal to elastic energy stored in the opossum shrimp.

$$
m g h \approx \frac{1}{2} k \varepsilon^{2}
$$




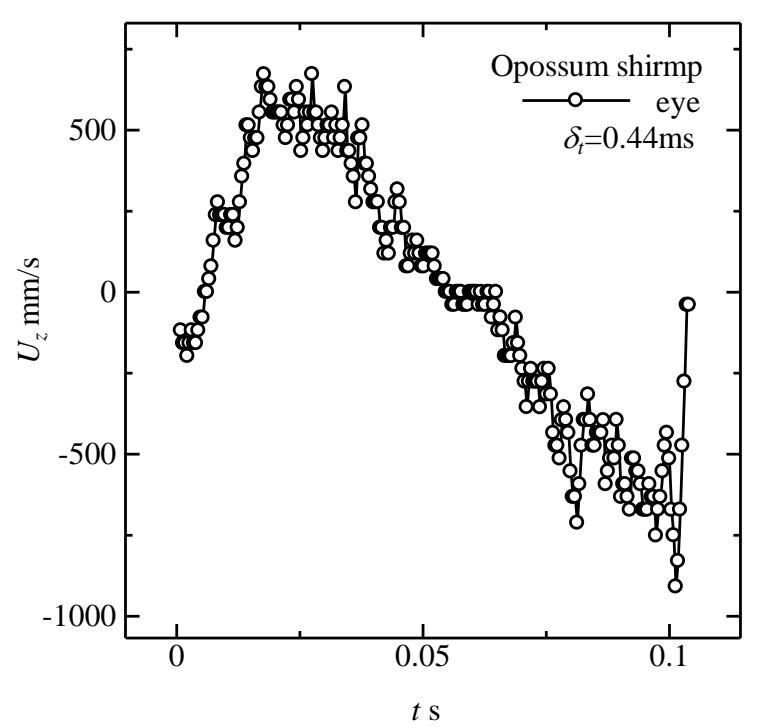

Fig. 10. Velocity variation of opossum shrimp motion during jump.

where $h$ is the maximum jump height, $g$ is the gravitational acceleration, $k$ is the spring constant, and $\varepsilon$ is the displacement. Since $h=16.3 \mathrm{~mm}$ and $m=5.9 \times 10^{-5} \mathrm{~kg}$ in Fig. 9, potential energy $E_{p}$ was calculated as $E_{p}=9.4 \times 10^{-6} \mathrm{~kg} \cdot \mathrm{m}^{2} \cdot \mathrm{s}^{-2}$ which approximates the kinetic energy generated by the opossum shrimp. Figure 10 shows the $\mathrm{z}$-component variation of opossum shrimp velocity during the jump motion in the air. It was defined that the upward direction of velocity was positive in Fig. 10. The negative velocity area in the early stage of jumping motion corresponds to the downward motion of the head by the bending of abdomen. The jump velocity shows the maximum value in the vicinity of $t=0.02 \mathrm{~s}$ in Fig.10. Afterwards, the velocity decreases, and the velocity is $U_{z}=0$ at the maximum height. Then the velocity of opossum shrimp motion increases in the negative direction with time. In the final stage, the opossum shrimp collides with the acrylic plastic plate.

The backward swimming of opossum shrimp by the fanning of the uropods is shown in Fig. 7. It can be seen from Fig. 9 that the opossum shrimp jumps by the similar uropods movement $(t=0-17.8 \mathrm{~ms}$ in Fig. 9). Figure 11 shows the angular velocity change in the fanning of the uropods. In Fig. 11, time $t$ corresponds to the time showed in Fig. 9. The mechanism of such a jump of opossum shrimp is not clear now. However, the abdomen bending and uropods movement generate jumping power. The force is generated, because the change in the angular velocity becomes an acceleration motion as shown in Fig. 11.

We can estimate the jumping force $F_{\text {jump }}$ of opossum shrimp using the mass $m=5.9 \times 10^{-5} \mathrm{~kg}$ and the mean acceleration $a=59.85 \mathrm{~m} / \mathrm{s}^{2}$ (see Fig. 10), that is, $F_{\text {jump }}$ $=3.5 \times 10^{-3} \mathrm{~N}$. On the other hand, we can estimate the thrust $T$ in opossum shrimp swimming using Eq. (1). The thrust $T$ was calculated as follows;

$$
T=6.9 \times 10^{-3} \mathrm{~N}
$$

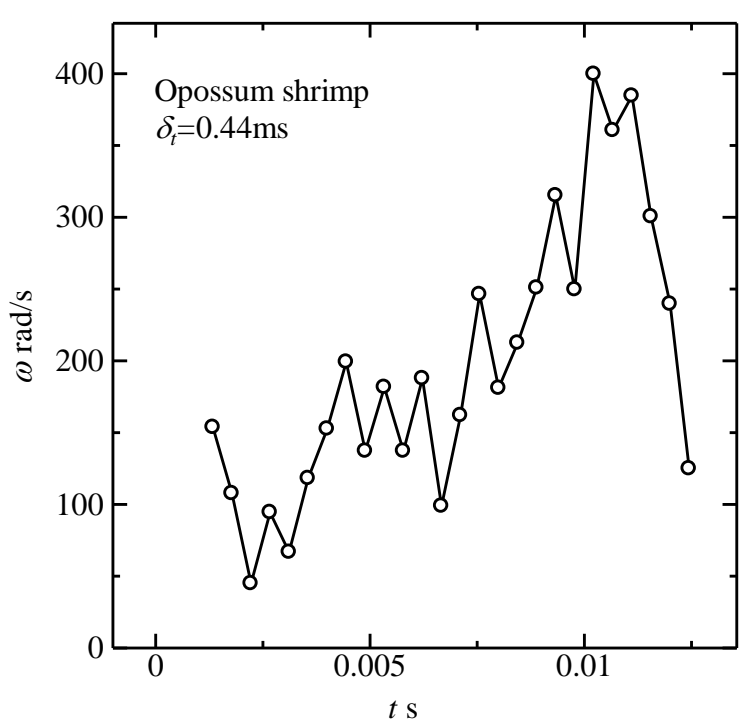

Fig. 11. Angular velocity variation of opossum shrimp uropod motion.

This value was calculated by substituting $\rho=1040$ $\mathrm{kg} / \mathrm{m}^{3}, \quad S=3.77 \times 10^{-6} \mathrm{~m}^{2}, \quad C_{D}=1.0, \quad U=0.49 \mathrm{~m} / \mathrm{s}$, $\alpha \rho V \approx m=5.9 \times 10^{-5} \mathrm{~kg}, d U / d t=54.63 \mathrm{~m} / \mathrm{s}^{2}$, into Eq.

(1). The calculations of jumping force and thrust force show $T>F_{\text {jump }}$. It was observed in the experiment that the opossum shrimp could jump more than the height $h=16.3 \mathrm{~mm}$ shown in Fig. 9 .

\section{CONCLUSIONS}

The dynamic behavior of opossum shrimp was studied with the digital high speed video camera system. The flow field around the tethered opossum shrimp was visualized. The results obtained are summarized as follows.

(1) Opossum shrimps possess two distinct forms of swimming behavior. The first is the behavior of cruise swimming. All of swimming legs reciprocate to produce thrust. The second form is the escape behavior. The thrust is produced by squeezing fluid from the gap created by the cephalothorax and the abdomen.

(2) Opossum shrimps can jump in the air. Opossum shrimps utilize a powerful tail-flip in jump motion.

(3) Visualization photographs of flow field around the tethered opossum shrimp show generation of the water flow to various directions.

\section{References}

[1] Azuma. A., The Biokinetics of Flying and Swimming, (1992), pp.1-265, Tokyo:Springer-Verlag.

[2] Dickinson, M. H., Farley,C. T., Full, R. J., Koehl, M.A.R., Kram, R. and Lehman, S., How Animals Move: An Integrative View, Science, Vol.288 (2000), pp.100-106.

[3] Sudo, S., Tsuyuki, K., Ito, Y. and Ikogai, T., A Study on the Surface Shape of Fish Scales, JSME International Journal, Vol. 45, No.4 (2002), pp.1100-1105.

[4] Sudo, S., Tsuyuki, K., Matsumoto, T., Yoshikawa, M. Watanabe, M. and Honda, T., Biomimetic Study of Diving Beetle Robot Propelled by Alternating Magnetic Field, International Journal of Applied Electromagnetics and Mechanics, Vol. 25, No.1 (2007), pp.601-606. 
JOURNAL OF AERO AQUA BIO-MECHANISMS, VOL.1, NO.1

[5] Sudo, S., Sekine, K., Shimizu, M., Shida, S., Yano, T. and Tanaka, Y., Basic Study on Swimming of Small Aquatic Creatures, Journal of Biomechanical Science and Engineering, Vol. 4, No.1 (2009), pp.23-36.
[6] Daniel, T. L. and Meyhöfer, E., Size Limits in Escape Locomotion of Carridean Shrimp Journal of Experimental Biology, Vol. 143(1989), pp.245-265. 